September 1999 • NREL/CP-520-27056

\title{
Magnetoresistance Technique for Determining Cross-Plane Mobility in Superlattice Devices
}

S.W. Johnston, R.K. Ahrenkiel, and D. Young National Renewable Energy Laboratory

R. Venkatasubramanian

Research Triangle Institute

Presented at the $18^{\text {th }}$ International Conference on Thermoelectrics

Baltimore, Maryland

August 29-September 2, 1999
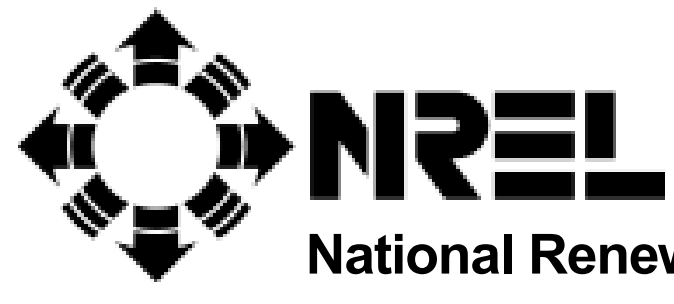

National Renewable Energy Laboratory

1617 Cole Boulevard

Golden, Colorado 80401-3393

NREL is a U.S. Department of Energy Laboratory

Operated by Midwest Research Institute $\bullet$ Battelle $\bullet$ Bechtel

Contract No. DE-AC36-98-G010337 


\section{NOTICE}

This report was prepared as an account of work sponsored by an agency of the United States government. Neither the United States government nor any agency thereof, nor any of their employees, makes any warranty, express or implied, or assumes any legal liability or responsibility for the accuracy, completeness, or usefulness of any information, apparatus, product, or process disclosed, or represents that its use would not infringe privately owned rights. Reference herein to any specific commercial product, process, or service by trade name, trademark, manufacturer, or otherwise does not necessarily constitute or imply its endorsement, recommendation, or favoring by the United States government or any agency thereof. The views and opinions of authors expressed herein do not necessarily state or reflect those of the United States government or any agency thereof.

Available to DOE and DOE contractors from:

Office of Scientific and Technical Information (OSTI)

P.O. Box 62

Oak Ridge, TN 37831

Prices available by calling 423-576-8401

Available to the public from:

National Technical Information Service (NTIS)

U.S. Department of Commerce

5285 Port Royal Road

Springfield, VA 22161

$703-605-6000$ or $800-553-6847$

or

DOE Information Bridge

http://www.doe.gov/bridge/home.html

Printed on paper containing at least $50 \%$ wastepaper, including $20 \%$ postconsumer waste 


\title{
Magnetoresistance Technique for Determining Cross-Plane Mobility in Superlattice Devices
}

\author{
S. W. Johnston, R. K. Ahrenkiel, D. Young, and R. Venkatasubramanian* \\ National Renewable Energy Laboratory, Golden, CO, USA \\ *Research Triangle Institute, Research Triangle Park, NC, USA
}

\begin{abstract}
The cross-plane mobility, in the direction perpendicular to the planes of a superlattice, is critical for the computation of the figure of merit (ZT) in a thermoelectric device. The measurement of cross-plane mobilities in thermoelectric superlattice structures cannot be performed by conventional techniques such as the van der Pauw method. Therefore, alternative techniques must be used to obtain this important parameter. Magnetoresistance is the increase in material resistivity due to a lengthened path for charge carriers in a perpendicular magnetic field. The magnetoresistance is related to the magnetic field strength as $(\mu \mathrm{B})^{2}$ in the standard configuration, but the field dependence is also influenced by device geometry. This work focuses on measuring superlattice samples of composition $\mathrm{Bi}_{2} \mathrm{Te}_{3} / \mathrm{Sb}_{2} \mathrm{Te}_{3}$ that are removed from their growth substrate and mounted on metal-coated substrates. This resulting mesa structure has a $100-\mu \mathrm{m}$-square contact metallization. Technical issues related to the sample preparation for the measurement are discussed. The magnetoresistance effect is expected to be small due to the anticipated low mobilities in $\mathrm{Bi}_{2} \mathrm{Te}_{3}$-based materials. Magnetoresistance studies with such superlattice thermo-elements were attempted using a dc magnetic field, but the sensitivity was insufficient. An ac magnetoresistance with lock-in detection can yield improved sensitivity.
\end{abstract}

\section{Introduction}

Magnetoresistance is the increase in sample resistance due to the presence of a magnetic field. It may be useful for determining mobility when the sample structure does not permit van der Pauw contacts typically used for Hall-effect mobility measurements. The increased resistance induced by an applied magnetic field is due to both resistivity changes in the semiconductor sample and geometrical effects. First, the physical magnetoresistance effect (PMR) is related to conduction-process anisotropy, multi-carrier-type conduction, and energy-dependent carrier scattering. [1,2] If the sample is a long, thin rectangular bar with an applied transverse magnetic field, Lorentz forces on the carriers are balanced by the Hall electric field developed across the bar. PMR occurs when carriers with energy above and below the average energy are under- and over-compensated by this Hall field and result in increased resistivity. [2] If the sample consists of a thin plate with metallic contacts on the large faces, then the Hall field due to the transverse magnetic field would effectively be shorted out by the contacts. With no Hall field, the current flows at the Hall angle and magnetoresistance is observed even with no PMR. This effect is geomagnetoresistance (GMR), and it can be a much larger effect than PMR. [2]

Long, rectangular samples with contacts at the ends of the long sample have a large length-to-width ratio $(\mathrm{L} / \mathrm{W})$, and the magnetoresistance effect is very small. [1] As L/W approaches zero, as it is for the Corbino disk, the magnetoresistance effect increases. [1] The resistance ratio of sample resistance in a magnetic field, $R_{B}$, to $R_{O}(B=0)$ is given by

$$
\frac{R_{B}}{R_{O}}=\frac{\rho_{B}}{\rho_{O}}\left\lfloor 1+\left(\mu_{G M R} B\right)^{2}\left(1-0.54 \frac{L}{W}\right)\right\rfloor
$$

where $\rho_{\mathrm{B}}$ is sample resistivity in a magnetic field, $\rho_{\mathrm{O}}$ is sample resistivity with no magnetic field, and B is magnetic field. [1] The geometric magnetoresistance mobility $\mu_{\mathrm{GMR}}$ is related to the Hall mobility $\mu_{\mathrm{H}}$ by

$$
\mu_{G M R}=\xi \mu_{H}
$$

where $\xi$ is the magnetoresistance scattering factor and varies from 1.00 to 1.25 depending on the type of scattering. [2]

The material to be measured is a superlattice structure consisting of alternating layers of p-type $\mathrm{Bi}_{2} \mathrm{Te}_{3}$ and $\mathrm{Sb}_{2} \mathrm{Te}_{3}$. Metal is deposited on the surfaces to provide ohmic contacts. The material is removed from its growth substrate and transferred to a conducting substrate, thus forming a mesa structure. The conducting substrate that the mesa sits atop is too soft to allow wire bonding. The superlattice has a thickness of approximately $1 \mu \mathrm{m}$ and a size of $100 \mu \mathrm{m}$ square. This gives an L/W ratio of around .01 which reduces Eq. 1 to the following. [1]

$$
\frac{R_{B}}{R_{O}}=\frac{\rho_{B}}{\rho_{O}}\left[1+\left(\mu_{G M R} B\right)^{2}\right]
$$

Initially, the magnetoresistance measurement was attempted using an apparatus capable of creating a $0.5 \mathrm{~T}$ dc magnetic field. A small sample holder was constructed for contacting the mesa structure while still fitting and attaching in the magnet core gap. The mobility was expected to be small, and the dc magnetoresistance data showed no statistical difference between resistance with the field on and that with it off due to signal noise and drift.

An ac measurement method can have many advantages. First, the modulation of sample current and magnetic field, each having an ac frequency, is known as a double ac measurement method. [3-10] The sample acts as a linear mixer, and the output signal is the product of current and magnetic field that has frequency components at the sum and difference frequencies. [3-10] With an applied current frequency of $f_{I}$ and a magnetic field frequency of $f_{B}$, the magnetoresistance signal is detected at $f_{I} \pm 2 f_{B}$ which is separated from other effects. [11] A selective linear ac amplifier is then used to reject all frequency components except the desired component. [5,7] Next, the use of lock-in detection increases noise rejection over dc measurements and thus increases measurement sensitivity. [3,10,12] Double ac measurement systems have been used for Hall measurements. [3-10] The alternating magnetic fields are usually limited to low frequencies due to coil inductance and high currents. [13] Also, with electromagnets, the magnetic field intensity decreases with increasing frequency. [9] Magnetic fields of 0.1 to $0.7 \mathrm{~T}$ have been generated at corresponding freqencies of $60 \mathrm{~Hz}$ down to 1.5 Hz. $[3-5,8,9,11]$ 


\section{Experimental Apparatus}

In a double ac measurement system, the sample current and the magnetic field are each periodic functions of time but have different frequencies. The magnetoresistance signal is proportional to $\mathrm{B}^{2}$; thus, a large field is desirable. Also, noise considerations favor a high frequency. Permanent rare earth magnets $(\mathrm{NdFeB})$ were chosen for their high field strength and potential to generate larger frequencies by mechanical movement. Twelve 7/8"-diameter-by-1/2"-thick disc magnets are mounted in a circular configuration (six to a side) where neighboring and opposing magnets have opposite poles (see Figure 1). Because magnetic flux lines follow the path of lowest reluctance, the gap between opposing magnets is made smaller than the spaces separating magnets on the same circular wheel. Thus, the flux is directed across the center gap. The wheel and axle are made of magnetic steel to complete the magnetic circuit. One complete revolution of the wheel creates three cycles of magnetic field. When spun by an 1800-rpm synchronous motor, the wheel creates an alternating magnetic field with a frequency of $90 \mathrm{~Hz}$. The measured peak-field strength is $0.86 \mathrm{~T}$. A pickup coil generates a voltage signal, as shown in Figure $2 \mathrm{a}$. This signal can then be integrated according to Faraday's Law to give the changing magnetic field with time, as shown in Figure $2 b$.

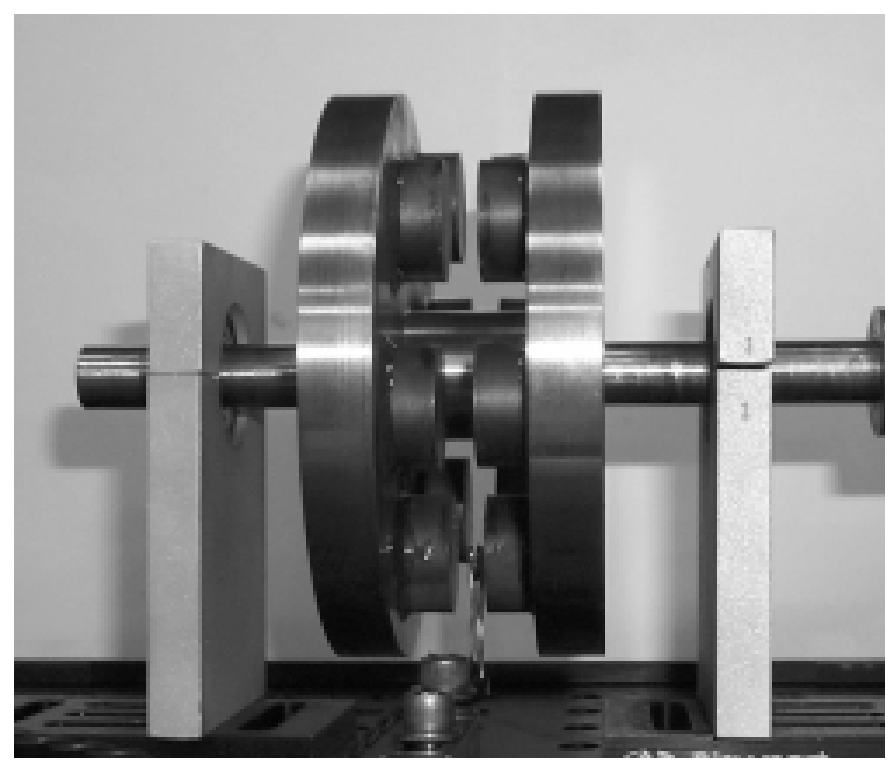

Figure 1. Permanent magnet spinning assembly.

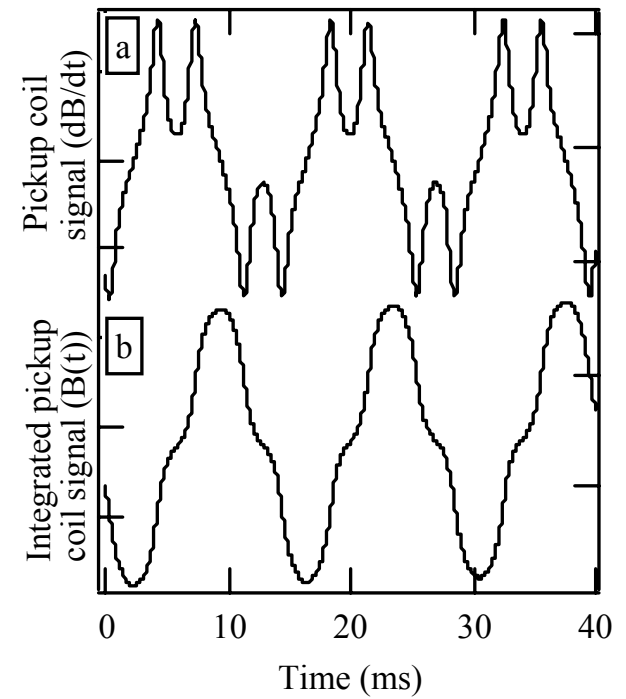

Figure 2. (a) Voltage signal from pickup coil. (b) Integrated signal showing changing magnetic field with time.

The magnetoresistance signal has the frequency of $f_{I} \pm 2 f_{B}$, as described previously. The $2 \mathrm{f}_{\mathrm{B}}$ component is generated by using a pickup coil that is positioned in the magnet assembly gap. This signal is initially integrated and then doubled using a diode doubly balanced mixer. After the mixer, the signal is then amplified and fed into a high-Q bandpass filter centered at $180 \mathrm{~Hz}$. This signal then becomes the reference to the lockin amplifier, as shown in the circuit-block diagram of Figure 3.

Figure 3 also shows the basis of electronics for driving current through the sample and measuring the sample magnetoresistance. A function generator is used to create a $10-\mathrm{kHz}$ signal, and this voltage is applied across the sample and a resistor in series. The sample current is then determined independently by measuring the voltage across the known series resistor. The voltage across the sample is directly proportional to the resistance of the sample as in a voltage divider circuit. The $10-\mathrm{kHz}$ voltage waveform across the sample results from the zero-magnetic-field resistance and contact resistance. The sample magnetoresistance modulates this voltage with a $2 \mathrm{f}_{\mathrm{B}}(180-\mathrm{Hz})$ signal that is proportional to magnetic field and the sample mobility, resistance, and current. The contact resistance is relatively independent of magnetic field. [1]

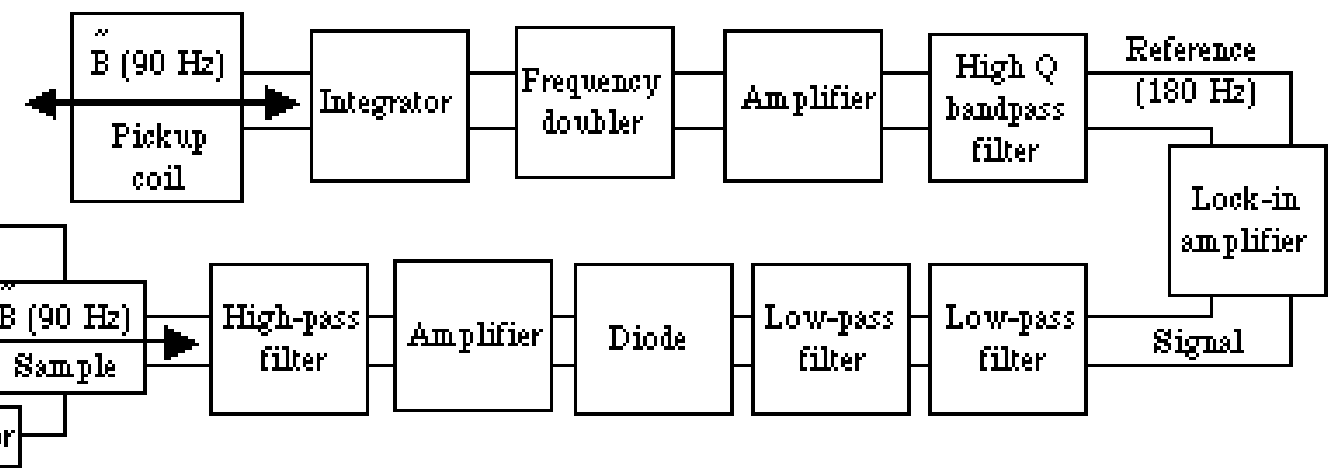

Figure 3. Circuit-block diagram for the double ac magnetoresistance measurement system. 
The probes used to contact the sample consist of a pair of twisted wires that do not spread apart until very near the sample. This minimizes the area of the closed-loop voltage probes and reduces the induced pickup voltage on the measurement circuitry due to the changing magnetic field. Fourier transform of the induced voltage shows significant frequency components as high as $1 \mathrm{kHz}$. This noise adds to the output signal but does not modulate it. It is filtered out as the signal is first fed into a highpass filter.

The voltage signal across the sample contains two components. First, the carrier frequency of $10 \mathrm{kHz}$ is due to the total sample resistance and sample current. Second, the amplitude modulation at $180 \mathrm{~Hz}$ is due to the magnetoresistance. This signal is amplified so that the peak values have a high enough voltage to forward bias a diode, approximately $0.7 \mathrm{~V}$. Then, by applying the signal across a diode, the carrier frequency is stripped away, leaving the modulation signal. This signal is smoothed by two stages of lowpass filters, which reduce ripple from the carrier frequency. This signal is then input into a lock-in amplifier, and along with the reference signal, the magnetoresistance effect can be detected.

\section{Experimental Results}

Two GaAs samples (one each of n-type and p-type) were measured using the spinning permanent magnet apparatus. These samples were chosen for initial measurement because of their relatively large size (for ease of contacting) and relatively high mobility. The n-type sample is a piece of GaAs wafer that is $4 \mathrm{~mm}$ by $4.5 \mathrm{~mm}$ and $380 \mu \mathrm{m}$ thick. The p-type piece is $3 \mathrm{~mm}$ by $5 \mathrm{~mm}$ and $480 \mu \mathrm{m}$ thick. Doped-indium contacts were made to the corners of each sample. A BIORAD HL5500 Hall System, used to measure the mobilities, showed 1280 and $82 \mathrm{~cm}^{2} / \mathrm{Vs}$ for the $\mathrm{n}$ - and p-type samples, respectively. The zero-field resistance of each sample was measured separately using four probes and two contacts, the same two contacts that were used for magnetoresistance. The resistances were 0.047 and $0.25 \Omega$ for the $n$ - and p-type samples, respectively. Because the L/W ratios of the samples are large (near 1), the magnetoresistance effect is expected to be small. [1] The magnetoresistance signal from the lock-in amplifier is plotted against sample current for each sample in Figure 4.

The n-type sample shows a larger magnetoresistance effect, even when having a smaller resistance. This is most likely due to the n-type sample's higher mobility. Because the sample geometry is not favorable for GMR, the magnetoresistance effect may not be proportional to $(\mu \mathrm{B})^{2}$, and may also depend on various PMR factors such as carriertype conductivity and scattering. Comparing the measured signals, the ratio of n-type to p-type sample response is 7.1. Taking into account the resistance ratio of $\mathrm{n}$ - to p-type being 0.19 , the magnetoresistance-effect ratio is then 38 . If the sample geometry favored GMR and the relation to $(\mu \mathrm{B})^{2}$ existed, then the mobility ratio would be $\sqrt{38}$, or 6.2 . The measured Hall mobility ratio is actually 15.6 , which lies between a $(\mu \mathrm{B})^{2}$ relation and a linear relation. $\mathrm{A}(\mu \mathrm{B})^{1.3}$ relation is calculated in order to equate the measured Hall and magnetoresistance mobilities for the GaAs samples.

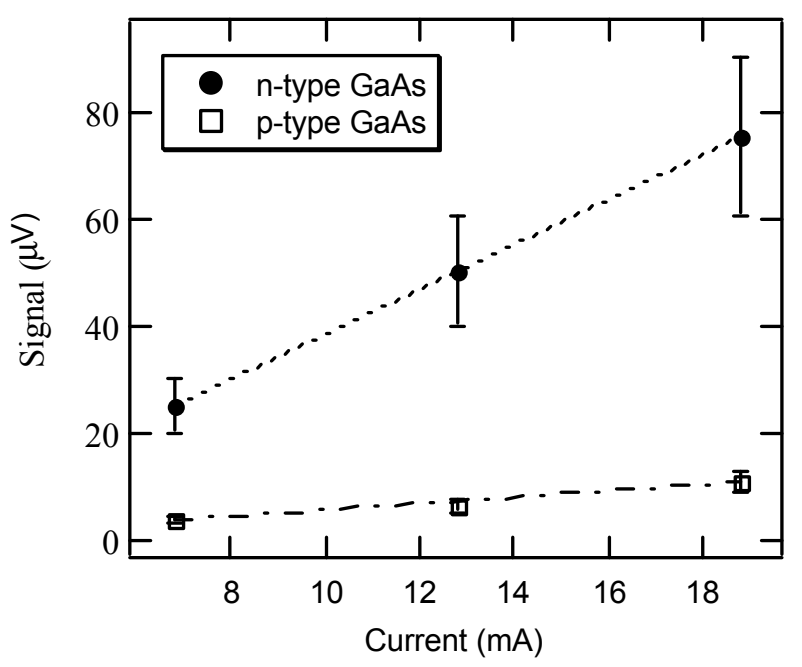

Figure 4. Output signals from the lock-in amplifier with reference signal at $180 \mathrm{~Hz}$ for the GaAs $\mathrm{n}$ - and p-type samples.

The superlattice mesa structure has a much more favorable geometry for GMR measurement; this sample was measured next. The difficulty of measuring this sample relates to the sample's small size--contact dimensions of $100 \mu \mathrm{m}$. The probe was modified by soldering fine gold ribbon wire to the probe tips. A four-point probe measurement to attain the sample resistance has not yet been successfully performed. However, the placement of two probes for the top and bottom contact of the mesas was accomplished, and the magnetoresistance-effect data are shown in Figure 5.

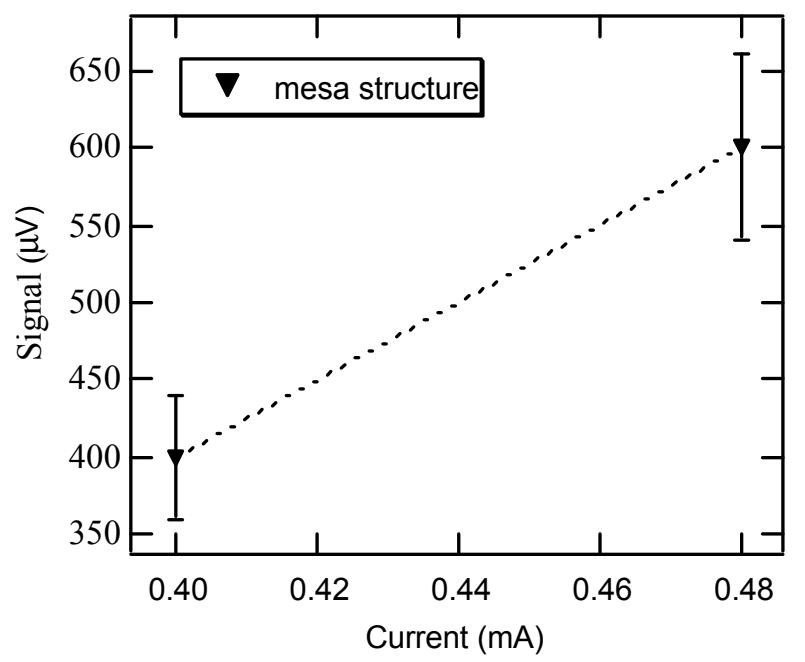

Figure 5. Output signal from the lock-in amplifier with reference signal at $180 \mathrm{~Hz}$ for the superlattice mesa sample.

Expected to have mobilities near or slightly better than the p-type GaAs sample, the mesa sample has a larger signal, which is mostly assumed to be related to the GMR effect and $(\mu \mathrm{B})^{2}$ relation. However, without $\mathrm{R}_{\mathrm{O}}$ resistance data or similar structures with which to compare data, the above results only show the measurement potential and a need for more samples and further technique refinement and development. 


\section{Discussion}

There are several issues to be addressed in the continuation of this project. Beginning with the measured data, characterization of the system is necessary for quantifying mobility. One idea is to fabricate GaAs samples with known mobility into similar mesa structures. Also, such samples may be measurable by both Hall and magnetoresistance for comparison. Next, the electronics are susceptible to noise, as they are mounted on a breadboard. Enclosing the electronics in a shielded and more stable environment should improve signal stability. The use of better performance amplifier electronics may also increase signal sensitivity. Also, an alternate method for signal demodulation would be to use a commercially available mixer. Finally, vibration from the magnet-spinning motor can disturb the probe contact to the sample. System modifications are needed to better isolate the sample and probes from the vibration created by the motor and spinning-magnets assembly, especially for the small superlattice mesa samples.

\section{Conclusions}

The magnetoresistance effect may be used to obtain mobility data when sample structures are not of a geometry that allows Hall mobility measurement. The magnetoresistance is sample-geometry dependent, but is related to sample mobility, resistance, and strength of magnetic field. For a given sample, the magnetoresistance effect can best be detected using an ac technique in which the magnetic field is as large as possible. Sensitivity is also increased as the magnetic-field freqency is increased. Permanent magnets were mechanically rotated to give a $90-\mathrm{Hz}$ magnetic field with a peak value of $0.86 \mathrm{~T}$.

GaAs samples were measured and a magnetoresistance effect was detected, even though the sample geometry was not favorable for GMR. A superlattice mesa structure with a much more favorable GMR geometry was also measured and a magnetoresistance effect signal was detected. Further technique developments and improvements are necessary for quantification of sample mobility.

\section{Acknowledgments}

This research was supported in part by Defense Advanced Research Projects Agency.

\section{References}

1. Schroder, D.K., Semiconductor Material and Device Characterization, John Wiley \& Sons, Inc. (New York, 1998), pp. 528-531.

2. Jervis, T.R. and Johnson, E.F., "Geometrical Magnetoresistance and Hall Mobility in Gunn Effect Devices," Solid-State Electronics, Vol. 13 (1970), pp. 181-189.

3. Chu, P., Niki, S., Roach, J.W., and Wieder, H.H., "Simple, Inexpensive Double AC Hall Measurement System for Routine Semiconductor Characterization," Rev. Sci. Instrum., Vol. 58, No. 9 (1987), pp. 1764-1766.

4. Russell, B.R. and Wahlig, C., "A New Method for the Measurement of Hall Coefficients," Rev. Sci. Instrum., Vol. 21 (1950), pp. 1028-1029.

5. Ryan, F.M., "Rotating Sample Method for Measuring the Hall Mobility," Rev. Sci. Instrum., Vol. 33, No. 1 (1962), pp. 76-79.
6. Yahia, J. and Perluzzo, G., "A Two Frequency AC Hall Apparatus for Measurements in Metals," Rev. Sci. Instrum., Vol. 44, No. 3 (1973), pp. 335-337.

7. Heinemann, K., Krokoszinski, H.J., Langohr, K., and Barner, K., "Novel Hall-Effect Measuring Device for Use in Superconducting Solenoids," Rev. Sci. Instrum., Vol. 56, No. 1 (1985), pp. 136-141.

8. Kaneda, T., Kabayashi, S., and Shimoda, K., "A Double AC Method for Sensitive Measurement of the Hall Effect," Jpn. J. Appl. Phys., Vol. 12, No. 9 (1973), pp. 1335-1337.

9. Lupu, N.Z., Tallan, N.M., and Tannhauser, D.S., "Apparatus for Measuring the Hall Effect of LowMobility Samples at High Temperatures," Rev. Sci. Instrum., Vol. 38, No. 11 (1967), pp. 1658-1661.

10. McLevige, W. V., Chatterjee, P. K., and Streetman, B. G., "Versatile Double AC Hall Effect System for Profiling Impurities in Semiconductors," J. Phys. E, Vol. 10 (1977), pp. 335-337.

11. Lundberg, B. and Backstrom, G., "Hall Voltage and Magnetoresistance of Bi Measured by a Sum Frequency Method in a Belt Apparatus," Rev. Sci. Instrum., Vol. 43, No. 6 (1972), pp. 872-875.

12. Lavine, J.M., "Alternate Current Apparatus for Measuring the Ordinary Hall Coefficient of Ferromagnetic Metals and Semiconductors," Rev. Sci. Instrum., Vol. 29, No. 11 (1958), pp. 970-976.

13. Altwein, M., Finkenrath, H., and Stockel, T., "Low Temperature Hall Measurements in a Cryostat with a Built-in Magnet," J. Phys. E, Vol. 6 (1973), pp. 623-627. 